\title{
Simple and complex movements off and on treatment in patients with Parkinson's disease
}

\author{
R BENECKE,* J C ROTHWELL, J P R DICK, B L DAY, C D MARSDEN
}

From the University Department of Neurology, and Parkinson's Disease Society Research Centre, Institute of Psychiatry and King's College Hospital Medical School, London, UK

SUMmaRY The performance of a $15^{\circ}$ elbow flexion and a $30 \mathrm{~N}$ hand squeeze was studied in nine normal subjects and in five patients with Parkinson's disease. The patients were studied when immobile after 12-18 hours withdrawal from antiparkinsonian drug therapy (OFF) and when more mobile after drugs (ON). Subjects performed each movement separately (simple movements) and both movements either simultaneously or sequentially (complex movements). The duration of both movements and the time between the onset of each movement in the sequential task (interonset latency) were measured. The patients OFF therapy had longer movement times than normal for both movements; these improved after levodopa but still were not normal. When the patients performed complex movements there was an extra slowness, over and above that seen for simple movements, in both movements of the simultaneous task and in the second movement of the sequential task. In addition, the interonset latency in the sequential task was longer than normal. After levodopa there was a more striking improvement in the movement times of complex movements than there had been for simple movements. The interonset latency in the sequential task was shorter than before therapy though still was not the same as in normal subjects. It is suggested that the basal ganglia are not only involved in the execution of simple motor programmes, but also play some role in the superimposition and sequencing of motor programmes.

The performance of simple single joint movements in akinetic and bradykinetic patients with Parkinson's disease shows poor correlation with the overall mobility of the same individuals in everyday life. For example, Evarts et al $^{1}$ made a detailed study of reaction times, but found that this parameter was not a useful indicator of Parkinsonian akinesia and bradykinesia; they suggested that movement times might provide a more useful objective measure. Baroni et al, ${ }^{2}$ examined the effect of levodopa on velocity of arm abduction in a group of previously untreated patients with Parkinson's disease. Although patients improved when $\mathrm{ON}$ therapy, their movements still were slower than normal. In our own studies ${ }^{3}$ on wrist movement, more severely affected patients were studied taking drugs (ON) and 12-24 hrs after withdrawal (OFF) of their normal therapy. Again,

*Permanent address: Abteilung fur klinische Neurophysiologie, Zentrum fur Neurologische Medizin, Universitat Gottingen, West Germany.

Address for reprint requests: Prof CD Marsden, Institute of Psychiatry, De Crespigny Park, London SE5 8AF, UK.

Received 1 May 1986.

Accepted 5 June 1986 levodopa increased the speed of their wrist movements, but their performance never approached that found in age-matched normal subjects, even though there was a striking difference in the mobility of patients in the ON versus the OFF condition. The conclusion was that measurements of such a simple movement do not provide a good description of akinesia and bradykinesia or of clinical change in Parkinson's disease. We argued that there must be deficits in other aspects of motor performance which contribute to the great motor disability seen in patients OFF drug therapy.

In a series of recent investigations ${ }^{4-6}$ it has been shown that complex simultaneous and sequential movements are much more affected in patients with Parkinson's disease than simple single joint movements. In these studies the movements analysed were an elbow flexion and an isometric opposition of thumb and fingers. When patients with Parkinson's disease had to make both movements at the same time, there was an additional increase in movement times over and above that seen in the separate movements alone. Similarly there also was an additional slowness when both movements had to be performed in a sequence. 
Table 1 Clinical details of patients $O N$ and OFF normal drug therapy

\begin{tabular}{|c|c|c|c|c|c|}
\hline Patient & $A g e(y r)$ & $\begin{array}{l}\text { Akinesia* } \\
O F F / O N\end{array}$ & $\begin{array}{l}\text { Tremor* } \\
\text { OFF } / O N\end{array}$ & $\begin{array}{l}\text { Rigidity* } \\
O F F / O N\end{array}$ & $\begin{array}{l}\text { Time aftert last } \\
\text { medication }(h)\end{array}$ \\
\hline $\begin{array}{l}\text { 1-AG } \\
2-W R \\
3-A R \\
4-M C \\
5-A B\end{array}$ & $\begin{array}{l}67 \\
37 \\
55 \\
61 \\
52\end{array}$ & $\begin{array}{l}5 / 3 \\
3 / 1 \\
5 / 2 \\
2 / 1 \\
3 / 1\end{array}$ & $\begin{array}{l}1 / 0 \\
5 / 1 \\
0 / 0 \\
1 / 0 \\
5 / 1\end{array}$ & $\begin{array}{l}5 / 2 \\
3 / 0 \\
1 / 0 \\
1 / 0 \\
3 / 1\end{array}$ & $\begin{array}{l}12 / 1 \cdot 0 \\
10 / 0.5 \\
18 / 1 \cdot 0 \\
12 / 0.75 \\
10 / 0.5\end{array}$ \\
\hline
\end{tabular}

*Akinesia, tremor and rigidity were graded clinically on a scale from $0-5$ when $\mathrm{OFF}$ and $\mathrm{ON}$ therapy.

+ Patients were tested $10-18 \mathrm{~h}$ after last medication (OFF condition) and then again $0.5-1.0 \mathrm{~h}$ after their regular dose of levodopa (ON condition).

In the present investigation it will be shown that changes in the performance of such complex movements in patients with Parkinson's disease ON and OFF treatment are much greater than those of simple movements.

\section{Materials and methods}

Simple and complex movements were studied in five male patients with at least 4 years' history of idiopathic Parkinson's disease (mean age 54.4 years, range 37-67
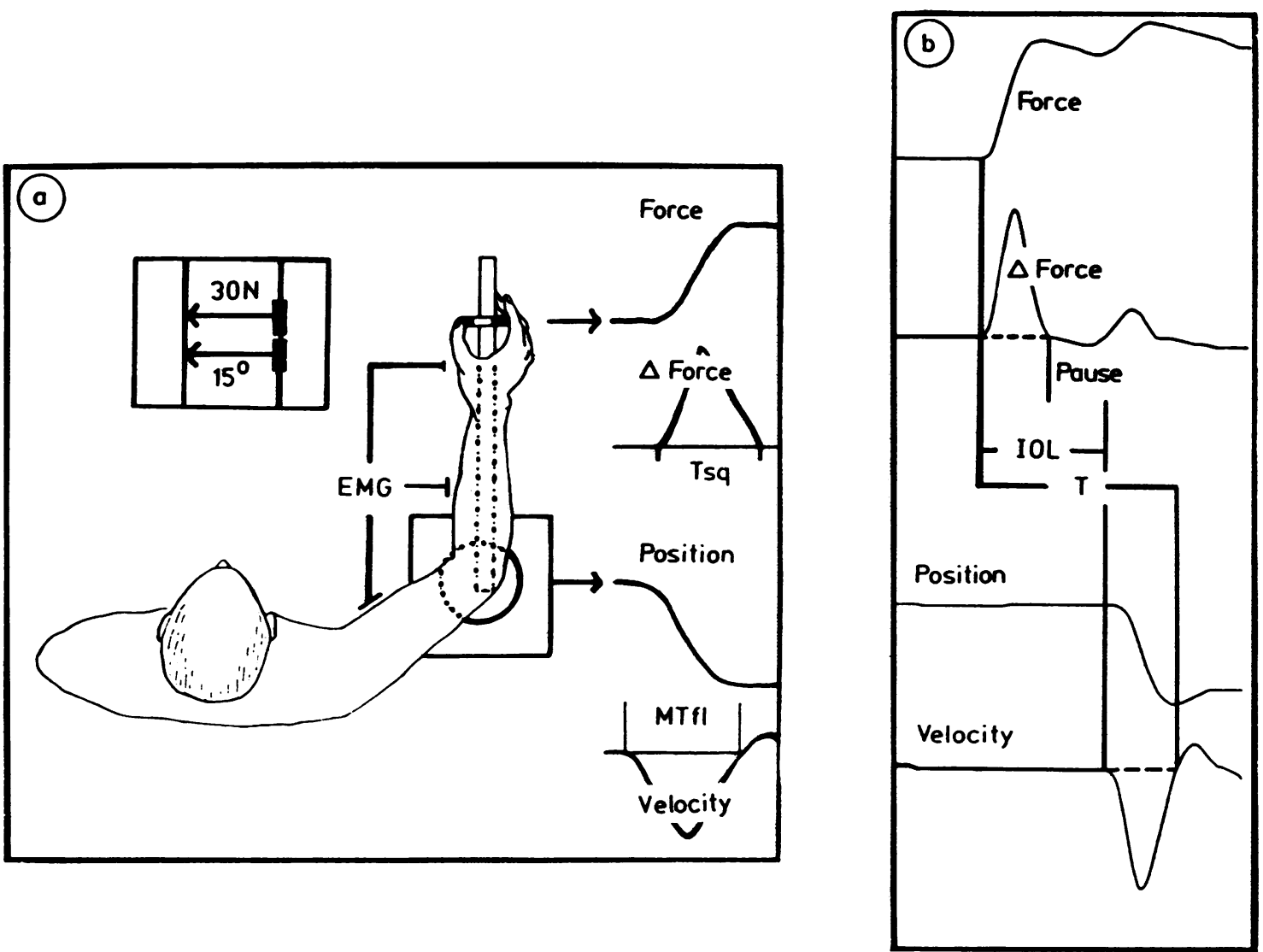

Fig 1 Schematic illustration of the experimental arrangements and measurements of movement performance. a shows the arrangement for the "squeeze" and "flex" tasks. Fixed starting and target positions (1 cm apart), as well as the movement response, were displayed as vertical bars on an oscilloscope screen $60 \mathrm{~cm}$ before the subject. Movement times (Tsqu, MTfl) were measured by means of $\Delta$ force (first derivative of the force signal) and velocity of "flex" (onset to zero crossing). b shows the measurements of mutual timing in the sequential "squeeze then flex" task. Total movement time (T) was measured from onset of "squeeze" to the termination of "flex". The interonset latency (IOL) was the time between the onsets of both movements. The pause was measured between termination of the first ("squeeze") to onset of the second movement ("flex"). 
years). All were right-handed and gave informed consent to the investigations. They had gained considerable benefit from levodopa therapy; the usual daily dose (with peripheral decarboxylase inhibitor) was $800-2,000 \mathrm{mg} / \mathrm{d}$. The patients were studied when akinetic $10-18 \mathrm{~h}$ after the last oral dose of levodopa (OFF) and then when mobile $30 \mathrm{~min}-1 \mathrm{~h}$ after administration of the drug $(\mathrm{ON})$. The motor functions of the patients (akinesia, tremor, rigidity) were assessed independently by two of the authors in the ON and OFF condition, using conventional clinical examination; a scale from 0-5 was used for each symptom (table 1). The electrophysiological tests of movement performance in the OFF and ON condition lasted each about $25 \mathrm{~min}$. The results in the patients were compared with those in a group of nine normal subjects (mean age 47.0 years; range 31 to 67 years).

Subjects were seated comfortably with the right arm abducted to $90^{\circ}$ at the shoulder. The semi-pronated forearm rested on a lightweight manipulandum which was pivoted so as to be co-axial with the elbow joint. At the end of the manipulandum, and adjusted according to the length of the forearm, was a "U"-shaped bar of aluminium which could be grasped between the thumb and fingers. A strain gauge was mounted on one of the vertical arms of the " $U$ " so that the force of squeeze could be monitored (fig 1).

Subjects were asked to perform four different tasks: (1). Flex the elbow joint as rapidly as possible in their own time through an angle of $15^{\circ}$ from a starting angle of $135^{\circ}$ ("flex" task). (2) Squeeze the strain gauge as rapidly as possible in their own time up to a force of $30 \mathrm{~N}$ ("squeeze" task). (3) Execute both tasks simultaneously as rapidly as possible in their own time ("both together" task). (4) Execute both tasks sequentially ("squeeze then flex" task) as rapidly as possible in their own time; the second movement ("flex") had to be initiated immediately after termination of the first ("squeeze"). The four tasks were performed in a cyclic order. Both elbow position and amount of grip force were displayed as two vertical bars $2 \mathrm{~cm}$ in length on an oscilloscope screen $60 \mathrm{~cm}$ before the subjects. Each individual performed about five practice trials of each of the four tasks; thereafter 10 single trials of each type were collected.

Electromyographic activity was recorded with surface electrodes over elbow flexors (biceps brachii, brachioradialis) and extensors (triceps), and finger muscles (opponens pollicis (opponens)). The angular position of the elbow joint was measured by a potentiometer mounted on the manipulandum, which was co-axial with the elbow joint. Joint position, joint velocity (electronically derived from the joint position signal), force and its first derivative (force), and rectified EMG signals were recorded by a PDP 12 computer with a sampling rate of $500 \mathrm{~Hz}$ per channel. The EMG signals were pre-amplified (Devices 3160 preamplifier with high and low pass filters set at $80 \mathrm{~Hz}$ and $2.5 \mathrm{kHz}(3 \mathrm{~dB}$ points) respectively), then amplified (Devices 3120 amplifier) and processed (Devices signal processor type 4010).

Measurements were made on each single record using the computer display unit. Duration of the elbow movement time (MTfl) was measured by visual inspection of the velocity signal. Force rise time (Tsqu) was measured from onset to zero crossing of $\Delta$-force (fig 1). In the sequential movements ("squeeze then flex") the interonset latency (IOL) was measured from onset of the "squeeze" ( $\Delta$-force) to onset of the "flex" (velocity signal). Total movement time for the sequential movement (T) was measured from onset of "squeeze" to termination of "flex" (first zero crossing of the velocity signal). Student's $t$ test was used to analyse the data; comparisons of patients with controls were analysed by unpaired $t$ tests; comparisons of patients ON and OFF were analysed by paired $t$ test.

Simple reaction times for an elbow flexion ("flex") were measured from the GO-signal (step-like jump of a vertical bar displayed on the oscilloscope) to movement onset (velocity for "flex"). A warning tone was given at randomised times $(2-5 \mathrm{~s})$ prior to the GO-signal. Excessively short $(<100 \mathrm{~ms})$ or long $(1.5 \times$ mean) response times were not included in the mean values.

\section{Results (table 2)}

\section{Simple movements}

Figure 2A shows the average performance in the simple elbow flexion task of a single representative patient in the OFF and $O N$ condition. Figure $2 B$ summarises the data from all five patients compared with those of normal subjects. The separate "flexion" movements (MTfl) were slower in the patients in the

Table 2 Summary of movement times in normal subjects and in patients $O N$ and $O F F$ therapy during performance of simple and complex tasks

(A) Durations of "squeeze" and "flex" movements in simple and complex tasks

\begin{tabular}{lllllll} 
& \multicolumn{1}{c}{ Tsqu } & & MTf & & \\
& Normal & OFF & ON & Normal & OFF & ON \\
\hline Separate & $156 \pm 23$ & $229 \pm 25$ & $210 \pm 15$ & $229 \pm 41$ & $379 \pm 100$ & $336 \pm 74$ \\
Simultaneous & $156 \pm 27$ & $330 \pm 62$ & $255 \pm 73$ & $216 \pm 38$ & $612 \pm 197$ & $449 \pm 114$ \\
Sequential & $150 \pm 25$ & $260 \pm 15$ & $239 \pm 27$ & $244 \pm 35$ & $490 \pm 140$ & $369 \pm 69$
\end{tabular}

(B) Details of performance in sequential "squeeze" then "flex" task

$I O L$

\begin{tabular}{lllllllll} 
Normal & OFF & ON & Normal & OFF & ON & Normal & OFF & ON \\
\hline $244 \pm 33$ & $441 \pm 47$ & $364 \pm 46$ & $94 \pm 28$ & $181 \pm 41$ & $125 \pm 26$ & $488 \pm 42$ & $932 \pm 178$ & $737 \pm 104$
\end{tabular}

Values are the mean ( \pm 1SD) movement times $(\mathrm{ms})$ of the nine normal subjects and the five patients with Parkinson's disease. Abbreviations as in fig 1 . 
(a)

Off

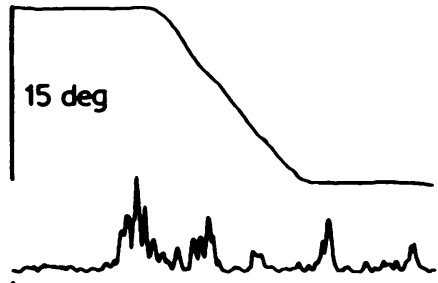

$1.0 \mathrm{mv}$

$\overline{200 \mathrm{~ms}}$
On

(b)

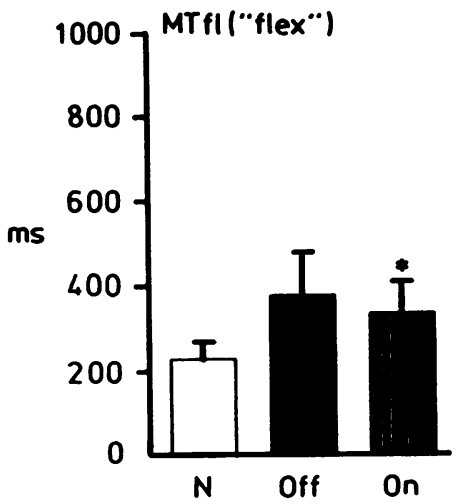

\section{Opponens}

Fig 2 Performance of simple separate "flex" task in OFF and $O N$ conditions of patients with Parkinson's disease and normal subjects. a shows representative single trials of elbow flexion $\left(15^{\circ}\right)$ in one patient in the OFF and the ON condition. Traces are elbow position (top), and rectified EMG signals (remaining traces). Calibrations for EMG signals (between second and third trace) apply to all muscles. Time and position calibrations in the left part (OFF) apply also to the right part (ON). b shows the mean values $( \pm I S D)$ of MTf in normal subjects $(n=9)$ (open column) and in all five patients $O N$ and $O F F$ therapy (shaded columns). There were significant differences between (I) performance of patients in the $O N$ and $O F F$ condition $\left(^{*}, p<0.05\right),(2)$ patients OFF versus normals $(p<0.01)$ and (3) patients $O N$ versus normals $(p<0.01)$.

(a)

Off

\section{$30 \mathrm{~N}$}

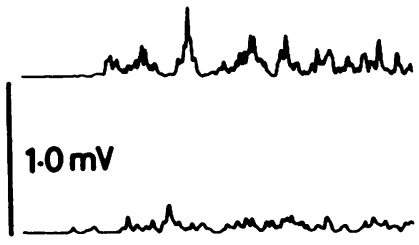

\section{$200 \mathrm{~ms}$}

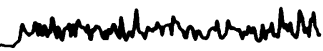

On
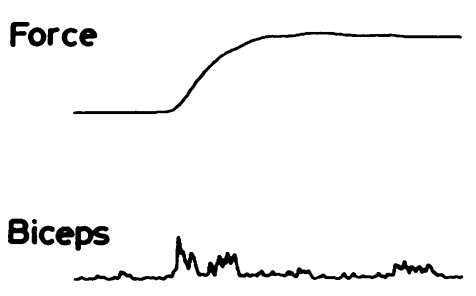

Triceps
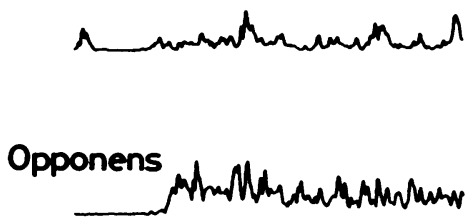

(b)

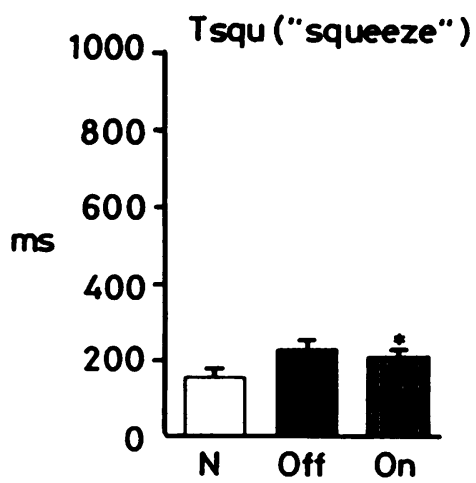

Fig 3 Performance of simple separate "squeeze" task in OFF and ON conditions of patients with Parkinson's disease and normal subjects. a show's representative single trials of "squeeze" to $30 \mathrm{~N}$ in one patient in the OFF and ON condition. Top traces are "squeeze" force; otherwise arrangements are as in fig 2. b shows the mean values ( \pm 1 SD) of Tsq in normal subjects $(n=9)$ (open column), and in the patients $(n=5)$ in OFF and ON (shaded columns) condition. There were significant differences in the performance of (1) patients in the ON and OFF condition $(*, p<0.05),(2)$ patients OFF versus normals $(p<0.001)$ and (3) patients $O N$ versus normals $(p<0.001)$. 
OFF condition than in normal subjects ( 379 vs. 229 ms; $p<0.01)$. When patients were ON therapy, their mean movement times (MTfl) decreased by $11.3 \%$ from 379 to $336 \mathrm{~ms}(\mathrm{p}<0.05)$. Despite the decrease in movement time in the patients when ON compared to OFF, their performance still was not as good as that of normal subjects; mean movement time in the patients ON was $336 \mathrm{~ms}$ compared to $229 \mathrm{~ms}$ in the normals $(\mathrm{p}<0.01)$.

Changes in the EMG patterns of simple elbow flexion were variable from patient to patient and trial to trial. In the OFF condition, all patients had double or multiple bursts of EMG activity in biceps and triceps. When $\mathrm{ON}$, the number of agonist bursts was reduced and the amplitudes of the first agonist burst were enhanced.

Figure 3 illustrates the performance in patients in the OFF and ON condition for a simple "squeeze". The duration of a simple isometric "squeeze" (Tsqu) was prolonged in the patients in the OFF condition when compared with the mean movement time in normal subjects ( 229 vs. $156 \mathrm{~ms} \mathrm{p}<0.001)$. Levodopa treatment improved the patients' performance: when patients were ON therapy, movement time for "squeeze" decreased by $8 \cdot 3 \%$ from 229 to $210 \mathrm{~ms}$ (p $<0.05$ ). However, their movement times still were longer than in normal subjects $(210$ vs. $156 \mathrm{~ms} \mathrm{p} \mathrm{<}$ 0.001 ).

The EMG patterns of the opponens during the squeeze in the OFF and ON condition showed a lack of phasic activity during the rise of force, which in normal subjects was always $100 \%$ of the subsequent tonic activity required to hold the force.

\section{Simultaneous movements}

As described in a previous investigation ${ }^{4} 5$ movement times increased still further in the patients when they were asked to perform "flex" and "squeeze" simultaneously, rather than separately. The pronounced slowness of both movements in the "both together" task is demonstrated for a representative patient with Parkinson's disease in the OFF and ON condition in fig 4A. The lower part of fig 4 summarises the data from all patients. When the patients were OFF therapy, their mean movement time for elbow flexion (MTf) was $379 \mathrm{~ms}$ when performed separately, but was $612 \mathrm{~ms}$ when performed at the same time as a "squeeze". Similarly the time taken for "squeeze" (Tsqu) rose from 229 (separate performance) to 330 ms (simultaneous performance) $(\mathrm{p}<0.01$ for both movements (paired $t$ test).

The EMG pattern in the "both together" task was not that expected from superimposition of behaviour in the separate tasks alone. If a patient had a preserved three burst EMG pattern in biceps and triceps during a separate "flex", this changed to a multiple (a)

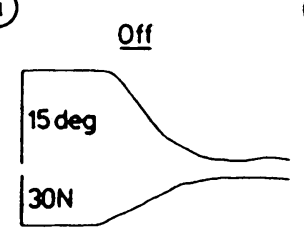

(b)
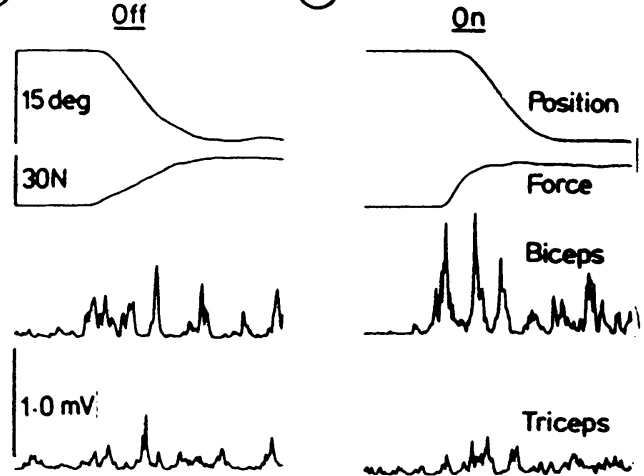

$\overline{200 \mathrm{~ms}}$
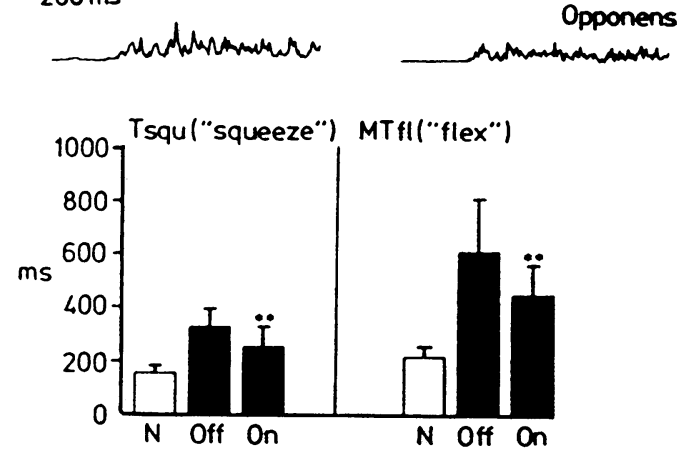

Fig 4 Performance of simultaneous "squeeze" and "flex" tasks. a shows representative single trials in a patient with Parkinson's disease in OFF and $O N$ condition. Traces are elbow position (top), "squeeze" force (second from top), and rectified EMG signals (remaining traces). For calibrations see legend of fig 2. b The histograms at the bottom show the mean values ( $\pm 1 S D)$ of MTfl and Tsqu in this task in normal subjects ( $n=9)$ (open columns) and in patients $(n=5)$ in the $O F F$ and $O N$ conditions (shaded columns). There were significant differences in both MTfl and Tsqu in (1) patients in the $O N$ and OFF conditions (**, $p<0.025$ for both), (2) patients OFF versus normals $(p<0.001$ for both) and (3) patients $O N$ versus normals $(p<0.01$ for Tsqu and $p<0.001$ for $M T f$ )

burst pattern or was replaced by tonic activity of biceps and triceps. Patients who already exhibited multiple bursts in the separate "flex" task changed to an exclusively tonic pattern in the "both together" task. The opponens muscle, recruited during "squeeze", showed a further decrease of the peak, or a slower rise of activity, rather than an abrupt increase in EMG, during the "both together" task.

When movement performance was compared in OFF and ON conditions, a pronounced improvement after drug administration was observed (fig 4). The mean movement time for "flex" (MTfl) decreased 
(a)

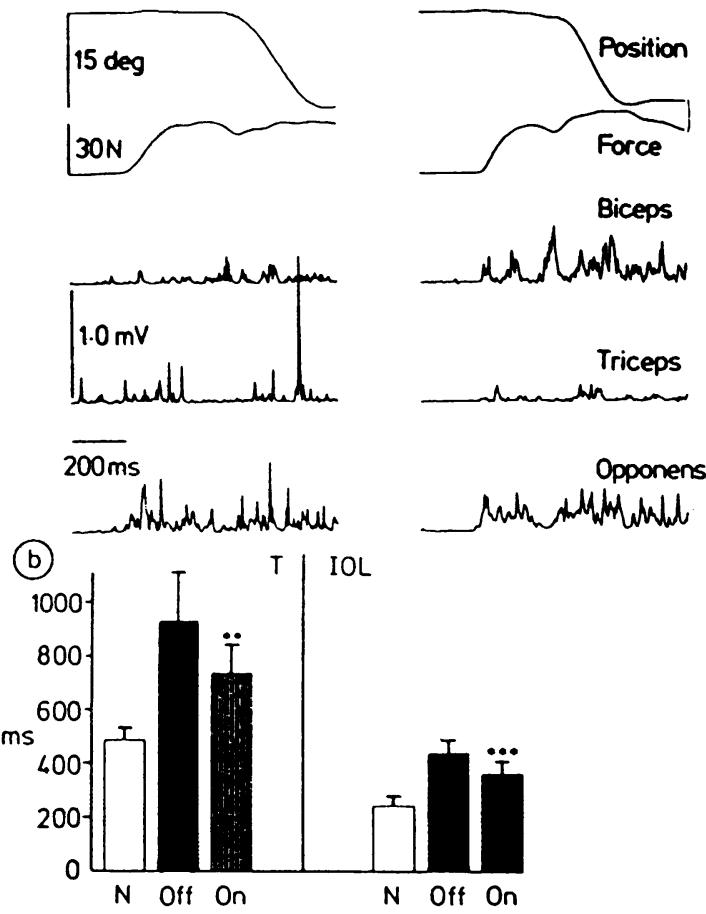

Fig 5 Performance of the sequential "squeeze then flex" task. a shows representative single trials in a patient with Parkinson's disease in OFF and ON condition. Traces from top to bottom and calibrations as in fig 4 . b shows the mean values $( \pm I S D)$ of total movement time $T$ (left) and interonset latency IOL (right) in normal subjects $(n=9)$ (open columns) and in patients $(n=5)$ in the ON and OFF conditions ( shaded columns). There was significant differences in both $T$ and IOL in (1) patients $O N$ and $O F F$ therapy $\left({ }^{* *}, p<0.025\right.$ for $T ;{ }^{* * *}, p<0.001$ for $\left.I O L\right)$, (2) patients OFF versus normals $(p<0.001$ for both $T$ and $I O L)$, and (3) patients $O N$ versus normals ( $p<0.01$ for both).

from 612 to $449 \mathrm{~ms}$, and for "squeeze" (Tsqu) from 330 to $255 \mathrm{~ms}$ ( $\mathrm{p}<0.025$ for both "flex" and "squeeze"). The percentage decreases amounted to 26.6 and $22.7 \%$, respectively. Movement performance in the ON condition, however, was still slower than that in normal subjects $(449 \mathrm{vs} .216 \mathrm{~ms}, \mathrm{p}$ $<0.01$ for "flex"; 225 vs. $156 \mathrm{~ms}, \mathrm{p}<0.001$ for "squeeze").

\section{Sequential movements}

Figure 5A shows the performance in the sequential "squeeze then flex" task in a representative patient with Parkinson's disease in the OFF and ON condition. Figure 5B summarises tile data from all patients. In patients with Parkinson's disease, movement times in the sequential task were prolonged, and their timing was disturbed. In the OFF condition, the mean time for the "squeeze" (Tsqu) alone was $229 \mathrm{~ms}$; when performed in a sequence with "flex" it was $260 \mathrm{~ms}$ ( $<<0.025)$. Mean time for the "flex" (MTfl) alone was $379 \mathrm{~ms}$; when performed after a "squeeze" it was $490 \mathrm{~ms}(\mathrm{p}<0.01)$. In the sequential movement mean Tsqu was longer in patients than normals ( 260 vs. 150 $\mathrm{ms}, \mathrm{p}<0.001)$, as was mean MTfl (490 ms vs. $244 \mathrm{~ms}$; $\mathrm{p}<0.01)$.

The mean interonset latency (from onset of "squeeze" to onset of "flex") in the OFF condition was prolonged when compared with that in normal subjects (441 ms vs $244 \mathrm{~ms} ; \mathrm{p}<0.001)$. The prolonged interonset latency was not simply the result of the slowed first movement; the pause between both movements of the sequence (from end of "squeeze" to onset of "flex") also was increased (181 ms vs $94 \mathrm{~ms}$; $p<0.001$ ). As a result of the prolongation in interonset latency and movement times (Tsqu; MTfl) the time to complete the entire sequence ( $T$ ) was strikingly prolonged in the patients as compared to normals $932 \mathrm{~ms}$ vs $488 \mathrm{~ms}$ ( $\mathrm{p}<0.001)$.

Comparison of the OFF and ON condition revealed pronounced drug effects on the performance of sequential movements. The mean interonset latency decreased by $17.5 \%$ from $441 \mathrm{~ms}$ (OFF) to $364 \mathrm{~ms}(\mathrm{ON})(\mathrm{p}<0.01)$ and the pause decreased by $31 \%$ from $181 \mathrm{~ms}$ (OFF) to $125 \mathrm{~ms}(\mathrm{ON})(\mathrm{p}<0.01)$. The time taken for the entire sequence $(T)$ was reduced by $20.9 \%$ from $932 \mathrm{~ms}$ (OFF) to $737 \mathrm{~ms}$ $(\mathrm{ON})(\mathrm{p}<0.025)$. The movement time for the first movement in the sequence (Tsqu) decreased from 260 ms (OFF) to $239 \mathrm{~ms}(\mathrm{ON})(\mathrm{p}<0.05)$; the movement time for the subsequent "flex" (MTf) showed a pronounced decrease from $490 \mathrm{~ms}$ to $369 \mathrm{~ms}$ ( $p<0.05$ ). The percentage decreases of Tsqu and MTfl amounted to $8.1 \%$ and $24.7 \%$, respectively.

Despite these beneficial effects of drug administration on performance in this sequential movement, the patients $\mathrm{ON}$ still were impaired compared with normal subjects (Tsqu: 239 vs $150 \mathrm{~ms}$; MTfl 369 vs 244; interonset latency 364 vs 244 ; pause 125 vs 94 ; total movement time T:737 vs $488 \mathrm{~ms}, \mathrm{p}<0.01$ ).

Simple reaction times for an elbow flexion in the ON condition were not different from those in the OFF condition $(208 \pm 33 \mathrm{~ms}$ (OFF) versus $208 \pm 35$ $\mathrm{ms}(\mathrm{ON})$. Reaction times in the patients also were not significantly prolonged compared to those in normals $(208 \pm 33 \mathrm{~ms}$, patients OFF vs $195 \pm 12 \mathrm{~ms}$, normal subjects; $\mathrm{p}>0.05$ ).

\section{Discussion}

The patients with Parkinson's disease OFF treatment generally performed much worse in the complex tasks 
of simultaneously or sequentially "squeezing" and "flexing", than in executing "squeeze" or "flex" alone as reported previously. ${ }^{4-6}$ The new results show that administration of levodopa to patients who had been withdrawn from therapy for the preceding 12-18 h also produced a more striking improvement in performance of complex combinations of two movements (generally more than $20 \%$ ) than it did in single movements executed alone (about $10 \%$ ). (The smaller improvement in Tsqu where a sequential movement of "squeeze then flex" was undertaken may reflect the fact that Tsqu, the first movement of the sequence, was not greatly prolonged compared to "squeeze" alone).

Why are simple one joint movements relatively less affected by levodopa than more complex tasks? Our hypothesis is that in order to combine movements such as "squeeze" and "flex" (either simultaneously or sequentially), one or more extra stages are added to the preparation or execution of the task. Previous studies $^{57}$ have shown that there is no correlation between the times taken to perform the individual components of "flex" and "squeeze" in a simultaneous or sequential movement. That is, in one movement, "flex" might be executed relatively fast and "squeeze" executed slowly: in the next "flex" might have been slow, and "squeeze" fast. The two component movements of the task appeared to be independent. Because of this we suggested that two separate motor programmes had been superimposed to produce the complex simultaneous movement of "squeeze and flex", and that the same two programmes had been run sequentially to produce the movement of "squeeze and flex". Superimposition or sequencing of motor programmes represents an extra stage in movement planning, over and above what is necessary to run each single programme.

Why was performance of patients with Parkinson's disease worse on complex simultaneous or sequential movements than on simple tasks? In the simultaneous task, the extra time required to complete the two movements reflected delay in completion of each component of the task, a delay not seen in normal subjects. In the sequential task, the extra time was due not only to the longer duration of each component movement (particularly of the second movement), but also to the longer pause between movements. Thus putting two programmes together, either simultaneously or sequentially, slowed both the execution of each individual component programme and their sequencing in Parkinson's disease. Levodopa not only improved the execution of each component programme, but also the speed of sequencing two sequential programmes. Accordingly, Parkinson's disease causes, and levodopa reverses, two defects in complex simultaneous and sequential movements not seen in simple one joint movements: (1) The extra slowness of each component movement (over and above that evident when the same movements are executed individually) and (2) The extra delay in sequencing two movements. Thus, the study of complex movements in Parkinson's disease brings out deficits not evident in simple single movements, and is therefore more likely to reflect general bradykinesia and akinesia.

It may turn out that analyses of motor tasks of even greater complexity in patients with Parkinson's disease will show even more pronounced abnormalities than of the "flex" and "squeeze" tasks employed in this study, and even greater differences in the OFF compared to the $\mathrm{ON}$ condition. For example, Schwab and colleagues ${ }^{8}$ demonstrated severe abnormalities in Parkinson's disease when patients were asked to trace the outline of a triangle with their right dominant arm, whilst squeezing the rubber bulb of a sphygmomanometer with their left arm. Patients not only were very slow at both tasks, but found it very difficult to execute them simultaneously; instead, they performed both tasks in sequence. Levodopa administration may lead not only to an increase of speed of execution of such movements, but also to recovery of the ability to perform such a complex action simultaneously.

The results of the present study suggest that the more complex a motor act, the greater the deficit in Parkinson's disease, and perhaps the correlation with clinical akinesia and bradykinesia. Parkinson's disease, despite the widespread nature of its pathology, especially in the later stages of the illness, is one of the best human models of disturbed basal ganglia function. Our results suggest that the basal ganglia are not only involved in the execution of simple motor programmes, but also play some role in the "higher level" superimposition or sequencing of two or more component programmes which make up a complex task.

Our thanks are due particularly to the five patients who agreed to be withdrawn temporarily from their normal drug therapy for the sake of this study. As always, Mr HC Bertoya and Mr R Miller provided indispensable design and technical information. RB was sponsored by the Deutsches Forschungesgemeinschaft; JCR is a Royal Society University Research Fellow. The work was funded by the Medical Research Council and the research funds of the Bethlem Royal and Maudsley Hospitals.

\section{References}

1 Evarts EV, Teravainen J, Calne DB. Reaction time in 
Parkinson's disease. Brain 1981;104:167-86.

2 Baroni A, Benuenuti F, Fantini L, Pantaleo T, Urbani F. Human ballistic arm abduction movements: effects of L-dopa treatment in Parkinson's disease. Neurology 1984;34:868-76.

3 Berardelli A, Dick JPR, Rothwell JC, Day BL, Marsden CD. Scaling of the size of the first agonist EMG burst during rapid movements in patients with Parkinson's disease. J Neurol Neurosurg Psychiatry 1986 (in press).

4 Benecke R, Day BL, Dick JPR, Marsden CD, Rothwell JC. Performance of various types of simultaneous movements in patients with Parkinson's disease. J Physiol (Lond) 1985;369:30P.
5 Benecke R, Rothwell JC, Dick JPR, Day BL, Marsden CD. Performance of simultaneous movements in patients with Parkinson's disease. Brain 1986;109:739-57.

6 Benecke R, Rothwell JC, Dick JPR, Day BL, Marsden CD. Performance of sequential movements in patients with Parkinson's disease. Brain 1986 (in press).

7 Benecke R, Rothwell JC, Day BL, Dick JPR, Marsden CD. Motor strategies involved in the performance of sequential movements. Exp Brain Res 1986;63:585-95.

8 Schwab RS, Chafez ME, Walker S. Control of two simultaneous voluntary motor acts in normals and in Parkinsonism. Arch Neurol Psychiatr 1954;72:591-8. 